\title{
The role of imitative mechanisms within the economic evolution $^{1}$
}

\author{
Agnieszka Lipieta ${ }^{2}$
}

\begin{abstract}
In the light of Schumpeter's pioneering vision of economic evolution the innovations and innovative entrepreneurs play a fundamental role in the economic development. However, imitations and producers-imitators are essential in diffusing and adapting innovations into the economic exchange processes. In this context the aim of the paper is to model and analyse some properties of imitative mechanisms appearing within the economic evolution.

Innovative and imitative mechanisms defined in Hurwicz's conceptual apparatus are analysed in the economy determined by the use of topological tools usually applied in the general equilibrium theory. As a result it is shown that, in the economy under study, imitative mechanisms are the reasons for and the consequences of innovative mechanisms as well as that the innovative and imitative processes can coexist in the framework of the same innovative mechanism. Moreover it is proven that under some assumptions equilibrium in the economic system analysed can be obtained as a consequence of either of an innovative or an imitative mechanism.
\end{abstract}

Key words: Schumpeterian evolution, innovation, imitation, private ownership economy, equilibrium.

JEL codes: D41, D50, O31.

\section{Introduction}

This paper is part of the research programme on modelling Schumpeter's economic evolution in the topological apparatus of the general equilibrium theory (see Arrow \& Debreu, 1954; Debreu, 1959; Lipieta \& Malawski, 2016, 2018; Ciałowicz \& Malawski, 2011, 2016). Changes in the economy can be observed due to the analysis of the so called innovative or imitative transformations

\footnotetext{
${ }^{1}$ Article received 16 April, accepted 24 September 2018.

The author acknowledges the support from research funds, granted to the Faculty of Finance and Law of the Cracow University of Economics, within the framework of the subsidy for the maintenance of research potential.

${ }^{2}$ Cracow University of Economics, Faculty of Finance and Law, Department of Mathematics, Rakowicka 27, Cracow, Poland, alipieta@uek.krakow.pl.
} 
of the area of production while economic mechanisms are modelled using the Hurwicz's apparatus of mechanism design theory (see i.e. Hurwicz, 1987; Hurwicz \& Reiter, 2006). Above all the paper aims at analysing the role of imitative mechanisms within the evolution of the economy.

It is shown that imitative processes do not disturb the innovative mechanisms as well as that these two processes can coexist in the framework of the same economic mechanism. It is also demonstrated that it is possible to obtain equilibrium in the economic system as the consequence of either an innovative or an imitative mechanism. The last result confirms the correctness of Schumpeter's (1912) and Shionoya's (2007) claims on the existence of processes resulting in equilibrium in the economy with respect to the economic system under consideration.

The paper consists of three sections. The first section deals with literature review. In the second section the private ownership economy as well as the mechanisms in the sense of Hurwicz are defined. The third section is devoted to the analysis of the role of imitation in the innovative processes. The paper is closed with conclusions.

\section{Literature review}

Imitation has been examined as a factor in economic development many times (see Schumpeter, 1912; Bessen \& Maskin, 2009; Glass, 2010; Mukoyama, 2003; Segestrom, 1990; Shenkar, 2010; Herrmann-Pillath, 2013; Safarzyńska \& van der Bergh, 2010). In many opinions there is even the statement that firms which imitate innovations and diffuse them throughout the economy play the central role in economic growth (Rossi, 2003).

In the light of Schumpeter's pioneering vision of economic evolution innovations and innovative entrepreneurs play an essential part in economic development. Joseph Schumpeter (1912), among others, wrote: "The fundamental impulse that acts and keeps the capitalistic engine in motion comes from the new consumers' goods, the new methods of production, the new forms of industrial organization that capitalist enterprise creates. (...) The opening up of new markets, foreign or domestic, and the organizational development (...) illustrate the same process of industrial mutation-if I may use that biological term-that revolutionizes the economic structure (...)." Similarly, Nelson and Winter (1982) wrote: "In our technical use of the term, "innovation" involves change in routine. (...) And the consequences of employing the innovation-changing the routine-in general will not be closely predictable until a reasonable amount of actual operating experience with it has been accumulated. (...). Innovations in organizational routine similarly consist, in large part, of new combinations of existing routines. An innovation may involve nothing more than the establishment of new patterns of information and material flows among 
existing subroutines." However, unlike Schumpeter, they used strict mathematical methodology for modelling economic development.

The role of innovation in the processes of economic growth were also stressed by Aghion and Howitt $(1992,1998)$. Their fundamental models of economic evolution, built with the use of differential equations, became the basis for the growth theory. Aghion and Howitt, differently from the Solow's neoclassical theory of economic growth in which the source of growth was seen in the accumulation of capital (see for instance Romer, 2012), claimed that the production of commodities of higher quality generated the growth of the economy.

Yet Shionoya indicated the existence of the mechanisms adapting innovations and directing the economic system towards equilibrium. Shionoya wrote (2007, p. 59): "Whatever destructive forces of innovation may emerge in the economy, markets can be relied on to adapt to them and absorb their effects in order to establish new equilibrium." As can be seen the importance of innovation in the economic process is fundamental.

However the part played by imitation in growth processes should not be neglected. Due to imitators, innovations are transferred into production processes. Imitation is an important mechanism underlying the behaviour of firms (Nelson \& Winter, 1982). On the other hand imitation can save on the costs of learning, inventing, experimenting, etc. on most of the expenses which are connected with innovative processes (Safarzyńska \& van den Bergh, 2010). Moreover the increases in the number and variety of imitations can intensify innovative changes throughout the production system (Ciałowicz \& Malawski, 2016).

\section{Model}

The definitions and the notions presented below are borrowed from (Hurwicz, 1987) and (Lipieta \& Malawski, 2018). Two sets of economic agents: consumers and producers, operating on the market with $\ell(\ell \in\{1,2, \ldots\})$ commodities, are considered. Linear space $\mathbb{R}^{\ell}$ with the standard scalar product

$$
(x \circ y)=\left(x_{1}, \ldots, x_{\ell}\right) \circ\left(y_{1}, \ldots, y_{\ell}\right)=\sum_{k=1}^{\ell} x_{k} \cdot y_{k},
$$

is the $\ell$-dimensional commodity space. It is denoted:

- $A=\left(a_{i}\right)_{i \in \mathbb{N}}-$ a countable set of consumers, $a_{i} \neq a_{j}$ if $i \neq j$,

- $B=\left(b_{j}\right)_{j \in \mathbb{N}}-$ a countable set of producers, $b_{i} \neq b_{j}$ if $i \neq j$,

- Pref $\subset \mathbb{R}^{\ell} \times \mathbb{R}^{\ell}$ is the family of all preference relations in $\mathbb{R}^{\ell}$.

For the given agents $(i, j \in \mathbb{N})$ the following characteristics are defined:

- $\chi: A \ni a_{i} \rightarrow \chi\left(a_{i}\right)=X^{a_{i}} \subset \mathbb{R}^{\ell}$ is a correspondence of consumptions sets satisfying

$\exists m \in \mathbb{N}$ such that $\forall i>m, \chi\left(a_{i}\right) \stackrel{\text { def }}{=}\{\mathbf{0}\}$, 
- $\epsilon: A \ni a_{i} \rightarrow \epsilon\left(a_{i}\right)=\epsilon^{a_{i}} \in X^{a_{i}}$ is the initial endowment mapping,

- $\varepsilon \subset A \times\left(\mathbb{R}^{\ell} \times \mathbb{R}^{\ell}\right)$ is a correspondence which to every consumer $a_{i} \in A$ assigns a preference relation $\preceq_{a_{i}}$ from set Pref restricted to set $\chi\left(a_{i}\right) \times \chi\left(a_{i}\right)$,

- $y: B \ni b_{j} \rightarrow Y^{b_{j}} \subset \mathbb{R}^{\ell}-$ a correspondence of production sets satisfying $\exists n \in \mathbb{N}$ such that $\forall j>n, y\left(b_{j}\right) \stackrel{\text { def }}{=}\{\mathbf{0}\}$.

A correspondence of production sets to every producer $b_{j}$ assigns a nonempty production set $y\left(b_{j}\right)=Y^{b_{j}} \subset \mathbb{R}^{\ell}$ of the producer's feasible production plans. Every production plan $y^{b_{j}} \in Y^{b_{j}}$ is identified with the production process of producer $b_{j}$ with outputs and inputs given by vector $y^{b_{j}} \in \mathbb{R}^{\ell}$.

The producer $b_{j}$ for which $y\left(b_{j}\right)=\{\mathbf{0}\}$ is called the inactive producer while the producer for which $y\left(b_{j}\right) \neq\{\mathbf{0}\}$ is called the active one. Similarly a correspondence of consumption sets to every consumer assigns $a_{i}$ a non-empty consumption set $\chi\left(a_{i}\right)=X\left(a_{i}\right)$ being a subset of the commodity space $\mathbb{R}^{\ell}$ and representing the consumer's feasible consumption plans. As in case of producers every consumption plan $x\left(a_{i}\right) \in X\left(a_{i}\right)$ is identified with the consumption process of consumer $a_{i}$ with outputs and inputs given by vector $x^{a_{i}} \in \mathbb{R}^{\ell}$. The consumer $a_{i}$ for which $\chi\left(a_{i}\right)=\{\mathbf{0}\}$ is called the inactive consumer while the consumer for which $\chi\left(a_{i}\right) \neq\{\mathbf{0}\}$ is called active.

In this research the prices of commodities are real numbers. Differently from Arrow and Debreu (1954, p. 8), it is not assumed that prices are nonnegative and normalized. The prices of the good are positive numbers for rare commodities, equal to zero for free goods, negative for harmful goods. In the third case an agent for which a harmful commodity is the output should pay. For example, industrial wastes can be harmful and their removal will require costs until the moment of the invention enabling their utilization and transformation into a desirable good. In Mas-Colell, Whinston and Green (1995, p. 20) the explanation for the consideration of negative prices is the following: "A negative price means that a "buyer" is actually paid to consume the commodity (which is not illogical for commodities that are "bad" such as pollution)". Hence in this research the space $\mathbb{R}^{\ell}$ is interpreted as the commodity-price space (see also Ciałowicz \& Malawski 2011, 2016; Lipieta \& Malawski 2016, 2018).

Let a price vector $p \in \mathbb{R}^{\ell}$ be given.

Definition 1. The two-range relational system $P_{q}=\left(B, \mathbb{R}^{\ell} ; y, p\right)$ is called the quasi-production system. If, additionally, for every $b_{j} \in B(j \in \mathbb{N})$,

$$
\eta^{b_{j}}(p) \stackrel{\text { def }}{=}\left\{y^{b_{j}^{*}} \in Y^{b_{j}}: p \circ y^{b_{j}^{*}}=\max \left\{p \circ y^{b_{j}}: y^{b_{j}} \in Y^{b_{j}}\right\}\right\} \neq \varnothing,
$$

then the quasi-production system $P_{q}$ is called the production system.

The elements $y^{b_{j}^{*}}$ of set $\eta^{b_{j}}(p)$ are called the optimal plans of producer $b_{j}$. In the spirit of the bounded rationality assumption (see Simon, 1955; Lipieta \& Malawski, 2016; Lipieta, 2013), in the quasi-production system, the aims of producers are not specified, while in the production system producers maxi- 
mize the profits at given prices and technologies. Similarly a quasi-consumption system is defined.

Definition 2. The three-range relational system $C_{q}=\left(A, \mathbb{R}^{\ell}, \operatorname{Pref} ; \chi, \epsilon, \varepsilon, p\right)$ is called the quasi-consumption system. If, for every $a_{i} \in A(i \in \mathbb{N})$,

$$
\begin{gathered}
\beta^{a_{i}}(p)=\left\{x \in \chi\left(a_{i}\right): p \circ x \leq p \circ \epsilon^{a_{i}}\right\} \neq \varnothing . \\
\varphi^{a_{i}}(p)=\left\{x^{a_{i}^{*}} \in \beta\left(a_{i}\right): \forall x^{a_{i}} \in \beta\left(a_{i}\right) x^{a_{i}} \preceq_{a_{i}} x^{a_{i}^{*}}, \preceq_{a_{i}} \in \operatorname{Pref}\right\} \neq \varnothing,
\end{gathered}
$$

then the quasi-consumption system $C_{q}$ is called the consumption system.

In the quasi-consumption systems the upper bound for a preference relation on a consumer's budget set does not have to exist. However it is assumed that every consumer realizes one of his optimal plans of action, if any exists.

On the basis of the above, assuming that consumers are the owners of firms and their shares in the firms' profits are given by the mapping $\theta: A \times \mathrm{B} \rightarrow[0,1]$ satisfying:

$$
\theta\left(a_{i}, b_{j}\right)=0 \quad \text { if } \quad i>m \quad \text { or } \quad j>n
$$

as well as

$$
\forall j \in\{1, \ldots, n\} \sum_{i=1}^{m} \theta\left(a_{i}, b_{j}\right)=1,
$$

the following definition is formulated (compare to Debreu, 1959; Mas-Colell et al., 1995):

Definition 3. The relational system $\varepsilon_{q}=\left(\mathbb{R}^{\ell}, P_{q}, C_{q}, \theta, \omega\right)$, where:

- $P_{q}=\left(B, \mathbb{R}^{\ell} ; y, p\right)$ is the quasi-production system,

- $C_{q}^{q}=\left(A, \mathbb{R}^{\ell}\right.$, Pref; $\left.\chi, \epsilon, \varepsilon, p\right)$ is the quasi-consumption system in which the budget set of every consumer $a_{i}$ is modified to the set

$$
\beta^{a_{i}}(p)=\left\{x \in X^{a_{i}}: p \circ x \leq w^{a_{i}}\right\}
$$

where

$$
w^{a_{i}}=p \circ \epsilon^{a_{i}}+\sum_{j=1}^{\infty} \theta\left(a_{i}, b_{j}\right) \cdot\left(p \circ y^{b_{j}}\right)
$$

is the wealth of consumer $a_{i}$ and vector $y^{b_{j}}$ denotes the plan of action of producer $b_{i}$

- $\epsilon\left(a_{i}\right) \stackrel{\text { def }}{=} 0 \in \mathbb{R}^{\ell}$ for $i>m$ as well as $\sum_{i \in \mathbb{N}} \epsilon^{a_{i}}=\omega$,

is called the private ownership economy with almost all inactive agents, in short the economy. If $P_{q}$ is the production system and $C_{q}$ is the consumption system 
then the private ownership economy with almost all inactive agents (also called the economy) is denoted by $\varepsilon_{q}$.

Note that in economy $\varepsilon_{q}$, for every $j \in \mathbb{N}$, the profit of each producer $b_{j}$, by realization of the plan $y^{b_{j}}$, is divided among all consumers according to function $\theta$. If, for every $i \in \mathbb{N}$,

- $\beta^{a_{i}}(p) \neq \varnothing$ and $\varphi^{a_{i}}(p) \neq \varnothing$, then consumer $a_{i}$ chooses one of his consumption plans $x^{a_{i^{*}}} \in \varphi^{a_{i}}(p) \subset X^{a_{i}}$ maximizing his preference on the budget $\operatorname{set} \beta^{a_{i}}(p)$,

- $\beta^{a_{i}}(p) \neq \varnothing$ and $\varphi^{a_{i}}(p)=\varnothing$, then a consumer $a_{i}$ chooses a consumption plan $x^{a_{i}} \in \beta^{a_{i}}(p)$, due to his own criterion,

- $\beta^{a_{i}}(p)=\varnothing$, then we assume that $x^{a_{i}}=0 \in \mathbb{R}^{\ell}$.

To every inactive consumer (for $i>m$ ) is assigned plan $x^{a_{i}}=0 \in \mathbb{R}^{\ell}$. The allocation $\left(\left(x^{a}\right),\left(y^{b}\right)\right)$, where $\left(x^{a}\right) \stackrel{\text { def }}{=}\left(x^{a_{1}}, \ldots, x^{a_{m}}, 0,0, \ldots\right)$ and $\left(y^{b}\right) \stackrel{\text { def }}{=}\left(y^{b_{1}}, \ldots, y^{b_{n}}, 0,0, \ldots\right)$ is called feasible, if

$$
\sum_{i \in \mathbb{N}} x^{a_{i}}-\sum_{j \in \mathbb{N}} y^{b_{j}}-\omega=0 .
$$

In economy $\varepsilon_{q}$, every agent realizes his optimal plan, namely every produc-

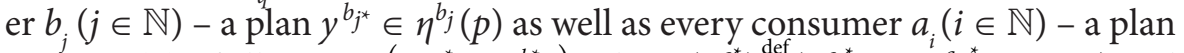
$x^{a_{i}{ }^{*}} \in \varphi^{a_{i}}(p)$. If allocation $\left(\left(x^{a^{*}}\right),\left(y^{b^{*}}\right)\right)$, where $\left(x^{a^{*}}\right) \stackrel{\text { def }}{=}\left(x^{a_{1}{ }^{*}}, \ldots, x^{a_{m^{*}}}, 0,0, \ldots\right)$ and $\left(y^{b^{*}}\right) \stackrel{\text { def }}{=}\left(y^{b_{1}{ }^{*}}, \ldots, y^{b_{n}{ }^{*}}, 0,0, \ldots\right)$, is feasible, then the sequence

$$
\left(\left(x^{a^{*}}\right),\left(y^{b^{*}}\right), p\right)
$$

is called the state of Walras equilibrium in economy $\varepsilon_{q}$.

The private ownership economy with almost all inactive agents is the static mathematical economic structure. To observe changes in the economy modelled in the above framework, the idea of economic mechanisms can be used (see also Lipieta \& Malawski, 2016). Let $E$ be the set of environments and $Z$ the set of outcomes.

Definition 4. (Hurwicz \& Reiter, 2006). The triple $\Gamma=(M, \mu, h)$, where

- $M \neq \varnothing$ is the message space,

- $\mu: E \rightarrow M$ is the message correspondence,

- $h: M \rightarrow Z$ is the outcome function,

is called the economic mechanism in the sense of Hurwicz.

The specification of the economic mechanism in the sense of Hurwicz should start from the determination of the so-called economic environment. The economic environment is the set of valuables and characteristics that determined individuals and firms as the economic agents in the economy under study. The space of messages $M$ is defined as the set all signals available to communications within the agents in the considered economic system, while 
the set of outcomes is the set of goals of the process modelled. The Hurwicz's economic mechanism is the relational system (see for instance Adamowicz \& Zbierski, 1997) consisting of:

- the message space $M$ that can be understood as the set of all feasible signals sent by economic agents; these signals are connected with agents' activities on the market and they are noticed, recognized and analyzed by other economic agents,

- the message correspondence $\mu$ which assigns to every environment, hence indirectly to economic entities, the signals (information) approved, which here means identified and analyzed, by economic agents,

- the outcome function $h$, which assigns to every message the outcome of activities of economic agents undertaken as a result of this message.

The economic mechanism in the sense of Hurwicz can be thought of as a system of exchanging and approving messages, which results in the agents' decisions concerning their activities on the markets. The method of defining the Hurwicz's mechanism relies on the determination of the economic environment depending on the initial conditions, specification and which are adequate for a given economic process, the space of messages as well as on the definition of the outcome function. More about economic mechanisms in the sense of Hurwicz can be found by the reader in (Hurwicz, 1987; Hurwicz \& Reiter, 2006; Lipieta \& Malawski, 2016, 2018).

The proposed approach significantly differs from the traditional models often quoted in literature (see Aghion \& Howitt, 1992, 1998; Romer, 2012; Acemoglu, 2009) used for studying the economic growth. Modelling the mechanisms of evolution in the Hurwicz's apparatus revealed the significant role of information and the way of exchanging it during innovative processes as well as, differently from Schumpeter's ideas, a large complexity of mechanisms appearing in the process of the evolution of the economy. Additionally diversification of modelled mechanisms demonstrates the complexity of economic processes and their results (outcomes). In the set of outcomes of innovative mechanism the effects of creative destruction are also revealed, besides new commodities, technologies and organizational structures, the old, unattractive products and technologies as well as the uncompetitive firms disappearing from the market in the new economic reality.

The mechanisms which result in innovative changes, differ not only in the economic environments, in the message spaces and in the sets of outcomes, but also in the sets of variables that characterize or will characterize the economic entities arising from the adoption by economic agents of innovative changes to their routine activities.

The axiomatization of mechanisms of Schumpeterian evolution can reveal the positive, from the producers' and consumers' points of view, qualitative properties of most of the examined mechanisms. Moreover it gives an opportunity to identify, in the set of possible mechanisms, the qualitative mecha- 
nisms with respect to the given set of agents, i.e. the mechanisms in which at least one agent from the given set would be better off due to a given criterion without making the rest of agents from this set worse off.

\section{Main results}

For observing, comparing and measuring changes and their results in the economy under consideration the idea of transformations of the systems presented is introduced (see Lipieta \& Malawski, 2016). Now some specific changes in the economy between two following time intervals are formalized. Let $t=0$ be an initial point of time of the modelling of a given economic process. Let $t, t^{\prime} \in\{0,1,2, \ldots\}, t<t^{\prime}$. If a quasi-production systems $P_{q}$ and $P_{q}^{\prime}$ are the mathematical equivalents of production sphere of an economy adequately at points of time $t$ and $t^{\prime}$, then components of system $P_{q}$ are transformed into the components of system $P_{q}^{\prime}$. In such a case the system $P_{q}^{\prime}$ is called the transformation of system $P_{q}$ that will be denoted by $P_{q} \subset P_{q}^{\prime}$. Every production plan $y^{b_{j}}(j \in \mathbb{N})$ is identified with a production process of producer $b_{j}$ realized at time $t$, where the amounts of inputs are given by negative coordinates of vector $y^{b_{j}}$, while the amounts of outputs are given by its add coordinates. Hence the vector $y^{b_{j}}$ describes technologies used in the given process. Assume that $\ell \leq \ell^{\prime}, B=B^{\prime}$ and $b \in B$ (the last means that there exists exactly one $j \in \mathbb{N}$ such that $b=b_{j}$ ).

Definition 5. A production plan $y^{\prime b} \in Y^{\prime b}$ is called:

1) the imitative plan, if

a) $\ell=\ell^{\prime} \Rightarrow \exists j \in \mathbb{N} y^{\prime b} \in Y^{b_{j}}$

b) $\ell<\ell^{\prime} \Rightarrow \exists j \in \mathbb{N} y^{\prime b} \in Y^{b_{j}} \times\{0\} \times \ldots \times\{0\} \subset \mathbb{R}^{\ell^{\prime}}$,

2) the innovative plan, if
a) $\ell=\ell^{\prime} \Rightarrow \forall j \in \mathbb{N} y^{\prime b} \notin \bigcup_{j \in \mathbb{N}} Y^{b_{j}}$,
b) $\ell<\ell^{\prime} \Rightarrow \forall j \in \mathbb{N} y^{\prime b} \notin \bigcup_{j \in \mathbb{N}}\left(Y^{b_{j}} \times\{0\} \times \ldots \times\{0\}\right) \subset \mathbb{R}^{\ell^{\prime}}$.

In an imitative production plan at time $t^{\prime}$ there are no new commodities with respect to time $t$ (conditions $1 \mathrm{a}$ and $1 \mathrm{~b}$ ). Additionally the old ones are not eliminated from the production process as well as the old technologies are still used. In an innovative production plan at time $t^{\prime}$ a new commodity (condition 2b) as well as a new technology can appear (conditions 2a and 2b) with respect to time $t$.

Definition 5 reflects the Schumpeter description of innovators and innovations as well as his understanding of imitators and imitations. According to Schumpeter (1964, p. 66) an innovator introduces new commodities, technologies, etc. on markets, while imitators follow an innovator and copy the previous solutions (Schumpeter, 1934, p. 133). That is why in this research 
all existing earlier goods, technologies, organizational structures and methods of production are called the imitations. Differently from Ciałowicz's and Malawski's (2016) definition of imitation extension producers' maximal profits are not taken into account as criteria in the division of goods into innovations and imitations in this paper.

It should be emphazed that, due to such modifications the division of the set of feasible production processes at time $t^{\prime}$ into two disjoint sets: the set of innovative processes with respect to time $t$ and the set of imitative processes with respect to time $t$ are obtained.

Definition 6. If $P_{q} \subset P_{q}^{\prime}$, then the system $P_{q}^{\prime}$ is called

- the imitative transformation of the system $P_{q}$, in short $P_{q} \subset_{i m} P_{q}^{\prime}$, if every production plan at time $t^{\prime}$ is the imitative plan with respect to time $t$,

- the innovative transformation of the system $P_{q}$, in short $P_{q} \subset_{i n} P_{q}^{\prime}$, if at least one production plan exists at time $t^{\prime}$ which is the innovative production plan with respect to time $t$.

The endogenous character of a production system in the private ownership economy leads to the conclusion that if $P_{q} \subset_{i m} P_{q}^{\prime}$, then $\ell=\ell^{\prime}$. In the imitative transformation of a given quasi-production system there appear neither new firms nor new commodities and the old ones are not eliminated from the production process (conditions 1a and 2b). The old technologies are also still used (condition 1b).

In the same way imitative and innovative consumption plans, as well as the imitative and innovative transformation of a quasi-consumption system can be defined. A producer (consumer), whose at least one feasible production plan is innovative is called the innovator, a producer (consumer), where every feasible plan of action is imitative is called the imitator. If $C_{q} \subset_{i m} C_{q}^{\prime}$, then the number of commodities $\ell$ and $\ell^{\prime}$ can be different; if $\ell<\ell^{\prime}$, then although producers-innovators introduce new commodities on the market, consumers are not interested in their consumption; if $\ell=\ell^{\prime}$ then new commodities do not appear on the market and consumers do not change their plans of action with respect to time $t$.

On the basis of the above the following is postulated:

Definition 7. Let $\varepsilon_{q} \subset \varepsilon_{q}^{\prime}$. Then

- the economy $\varepsilon_{q}^{\prime}$ is said to be the imitative transformation of economy $\varepsilon_{q^{\prime}}$, in short $\varepsilon_{q} \subset_{i m} \varepsilon_{q^{\prime}}^{\prime}$, if $P_{q} \subset_{i m} P_{q}^{\prime}$ and $C_{q} \subset_{i m} C_{q^{\prime}}^{\prime}$

- the economy $\varepsilon_{q}^{\prime}$ is said to be the innovative transformation of economy $\varepsilon_{q}$, in short $\varepsilon_{q} \subset_{i n} \varepsilon_{q^{\prime}}^{\prime}$ if $P_{q} \subset_{i n} P_{q}^{\prime}$.

Under the given assumptions the following is true:

Remark 1. Let $t, t^{\prime}, t^{\prime \prime} \in\{0,1,2, \ldots\}, t<t^{\prime}$ and $t^{\prime}<t^{\prime \prime}$. Let $\varepsilon_{q^{\prime}}, \varepsilon_{q}^{\prime}$ and $\varepsilon_{q}^{\prime \prime}$ be private ownership economies at points of time, adequately, $t, t^{\prime}$ and $t^{\prime \prime}$. Suppose 
that $\varepsilon_{q} \subset \varepsilon_{q}^{\prime}, \varepsilon_{q}^{\prime} \subset \varepsilon_{q}^{\prime \prime}$ as well as on time intervals $\left[t, t^{\prime}\right)$ and $\left[t^{\prime}, t^{\prime}\right)$ the components of economies, adequately $\varepsilon_{q}$ and $\varepsilon_{q}^{\prime}$ are constant. Then as the immediate consequence of Definitions 5,6 and 7 we get that if $\varepsilon_{q} \subset_{i m} \varepsilon_{q}^{\prime}$ and $\varepsilon_{q}^{\prime} \subset_{i m} \varepsilon_{q}^{\prime \prime}$, then $\mathcal{E}_{q} \subset_{i m} \mathcal{E}_{q}^{\prime \prime}$.

In Remark 1 it is inferred that an imitative transformation of an economy, which was also the imitative transformation of an initial economy, is also the imitative transformation of the same initial economy. Hence it can be stated that "being the imitative transformation" has the property of transitivity.

Notice also that, similarly as was done in (Lipieta, 2013, theorem 4.3), it can be proved that the transformation of the economic system considered from its one form into another is also the economic mechanism in the sense of Hurwicz. In such a mechanism:

- the characteristics of economic agents at time $t$ form the set of environments,

- the set of outcomes consists of the characteristics of economic agents at time $t^{\prime}$,

- the agents' activities on the market are identified as the messages sent by them,

- the set of messages is equal the set of outcomes $(M=Z)$,

- the outcome function is the identity function $\left(h=i d_{M}\right)$.

In a private ownership economy only the agents' present market activities are the source of information about their activities on competitive markets in future. New products and new technologies, which firms are going to introduce tomorrow, are hidden today. The mechanism of transformation can be identified with its results, namely with the production and consumption processes feasible at time $t^{\prime}$. By Definition 5 it is clear that imitative and innovative processes can coexist in a framework of a same mechanism as well as the fact that the existence of imitative processes do not disturb the innovative mechanisms. Moreover an imitative mechanism can precede and follow both the innovative and imitative mechanism.

In the next theorem a mechanism which, under some assumptions, transforms an initial economy $\varepsilon_{q}$, in which there is no equilibrium at given prices, into economy $\varepsilon_{q}^{\prime}$ which is in equilibrium at the same prices, is constructed. Recall that in economy $\varepsilon_{q}$, sets $\eta^{b_{j}}(p)$, for every $b_{j} \in B$, and $\varphi^{a_{i}}(p)$, for every $a_{i} \in A$, are not empty. Vector $p$ is not the equilibrium price vector, hence

$$
\omega \notin\left(\left(\varphi^{a_{1}}(p)+\ldots+\varphi^{a_{m}}(p)\right)-\left(\eta^{b_{1}}(p)+\ldots+\eta^{b_{n}}(p)\right)\right) .
$$

Let

$$
\zeta \stackrel{\text { def }}{=}\left(x^{a_{1}{ }^{*}}+\ldots+x^{a_{m}{ }^{*}}\right)-\left(y^{b_{1}^{*}}+\ldots+y^{b_{n}^{*}}\right)-\omega
$$

for some $x^{a_{1}{ }^{*}} \in \varphi^{a_{1}}(p), \ldots, x^{a_{m}{ }^{*}} \in \varphi^{a_{m}}(p), y^{b_{1}{ }^{*}} \in \eta^{b_{1}}(p), \ldots, y^{b_{n}{ }^{*}} \in \eta^{b_{n}}(p)$. 
Theorem 1. Let $t, t^{\prime} \in\{0,1,2, \ldots\}, t<t^{\prime}$. Let, at point $t, \varepsilon_{q}$ be a private ownership economy in which

$$
\forall i \in \mathbb{N} \forall a^{i} \in A: X^{a_{i}} \subset\left\{x \in \mathbb{R}^{\ell}: p \circ x=0\right\} .
$$

If

$$
p \circ \zeta \neq 0
$$

then there exist an economic mechanism in the sense of Hurwicz, which transforms, at point $t^{\prime}$, economy $\varepsilon_{q}$ into an economy $\varepsilon_{q}^{\prime}$, in which a state of equilibrium at price $p^{\prime}=p$ exists as well as $C_{q} \subset_{i m} C_{q}^{\prime}, \ell=\ell^{\prime}, n=n^{\prime}, \epsilon\left(a_{i}\right)=\epsilon^{\prime}\left(a_{i}\right)$ and $\varepsilon\left(a_{i}\right)=\varepsilon^{\prime}\left(a_{i}\right)$, for every $i \in \mathbb{N}$.

Proof. Firstly, notice that by assumption (4) vector

$$
g \stackrel{\text { def }}{=} \frac{p}{p \circ \zeta} \in \mathbb{R}^{\ell}
$$

satisfies the following system of two equalities:

$$
\left\{\begin{array}{c}
g \circ \zeta=1 \\
g \circ\left(x^{*}-\omega\right)=0 .
\end{array}\right.
$$

Hence mapping

$$
Q: \mathbb{R}^{\ell} \ni x \rightarrow x-(g \circ x) \cdot \zeta \in \mathbb{R}^{\ell}
$$

is the linear and continuous projection into space $\left\{x \in \mathbb{R}^{\ell}: p \circ x=0\right\}$ (see Cheney, 1966).

The environment of every agent $k \in K=A \cup B=\left(k_{i}\right)_{i \in \mathbb{N}}$ consisits of the extensions of characteristics defined separately in the production and in the consumption system of economy $\boldsymbol{\varepsilon}_{q}$. Precisely, the environment of every agent $k_{i} \in K(i \in \mathbb{N})$ at time $t$ is of the form:

$$
e^{k_{i}}=\left(Y^{k_{i}}(t), X^{k_{i}}(t), \epsilon^{k_{i}}(t), \varepsilon\left(k_{i}, t\right), \theta_{t}\left(k_{i}, \cdot\right)\right),
$$

where $Y^{k_{i}}(t)=Y^{k_{i}} \subset \mathbb{R}^{\ell}$ if $k_{i} \in B, Y^{k_{i}}(t)=\{0\}$ if $k_{i} \notin B, X^{k_{i}}(t)=X^{k_{i}} \subset \mathbb{R}^{\ell}$ if $k_{i} \in A$ as well as $X^{k_{i}}(t)=\{0\}$ if $k_{i} \notin A$. The extension of the initial endowment mapping is given by

$$
\epsilon^{k_{i}}(t)=\left\{\begin{array}{ccc}
\epsilon^{k_{i}} \in X^{k_{i}} & \text { if } & k_{i} \in A \\
0 \in \mathbb{R}^{\ell} & \text { if } & k_{i} \notin A .
\end{array}\right.
$$


Similarly the extension of the correspondence of preference relations at time is of the form:

$$
\varepsilon\left(k_{i} ; t\right)=\left\{\begin{array}{lll}
\preceq_{k_{i}}^{t} & \text { if } & k_{i} \in A \\
\{\varnothing\} & \text { if } & k_{i} \notin A
\end{array}\right.
$$

where $\varepsilon(\cdot ; t) \subset K \times\left(\mathbb{R}^{\ell} \times \mathbb{R}^{\ell}\right)$ and $\preccurlyeq_{k_{i}}^{t} \subset X^{k_{i}}(t) \times X^{k_{i}}(t)$ means the preference relationship of agent $k_{i}$ at time $t$. Additionally the mapping $\theta_{t}: K \times K \rightarrow[0,1]$, satisfying

- $\theta_{t}\left(k_{i}, \cdot\right) \equiv 0$ for $k_{i} \notin A$,

- $\theta_{t}\left(\cdot, k_{i}\right) \equiv 0$ for $k_{i} \notin B$,

- $\forall j \in\{1, \ldots, n\}: \sum_{i=1}^{m} \theta_{t}\left(a_{i}, b_{j}\right)=1$

is the share mapping at time $t$. Hence, the set of environments of agent $k_{i}$ at time $t$ is of the form:

$$
E^{k_{i}}=P\left(\mathbb{R}^{\ell}\right) \times P\left(\mathbb{R}^{\ell}\right) \times \mathbb{R}^{\ell} \times P\left(\mathbb{R}^{2 \ell}\right) \times \mathcal{F}(K,[0,1]),
$$

where $\mathcal{F}(K,[0,1])=\{f: K \rightarrow[0,1]\}$, while the set of environment at time $t$ is given by:

$$
E \stackrel{\text { def }}{=} E^{k_{1}} \times E^{k_{2}} \times \ldots
$$

The extended components of the economy at time $t^{\prime}$ are defined in the same way. Namely $Y^{k_{i}}\left(t^{\prime}\right)=Y^{\prime k_{i}} \subset \mathbb{R}^{\ell^{\prime}}$ for $k_{i} \in B, Y^{k_{i}}\left(t^{\prime}\right)=\{0\}$ for $k_{i} \notin B, X^{k_{i}}\left(t^{\prime}\right)=X^{\prime k_{i}} \subset \mathbb{R}^{\ell^{\prime}}$ for $k_{i} \in A, X^{k_{i}}\left(t^{\prime}\right)=\{0\}$ for $k_{i} \notin A$. Characteristics $\epsilon^{k_{i}}\left(t^{\prime}\right), \varepsilon\left(k_{i} ; t^{\prime}\right), \theta_{t^{\prime}}$ are also defined in the same way as earlier.

To determine the economic mechanisms the following is defined:

- $e=\left(e^{k_{1}}, e^{k_{2}}, \ldots\right)$, where, for every $k_{1}, k_{2}, \ldots \in K, e^{k_{i}}$ of the form (7),

- $M=\left\{\begin{array}{c}m=\left(m^{k_{1}}, m^{k_{2}}, \ldots\right): \forall i \in \mathbb{N} k_{i} \in K, \\ m^{k_{i}}=\left(p^{\prime}, y^{k_{i}}\left(t^{\prime}\right), x^{k_{i}}\left(t^{\prime}\right)\right) \wedge x^{k_{i}}\left(t^{\prime}\right) \in \varphi^{\prime k_{i}}\left(p^{\prime}\right) \wedge y^{k_{i}}\left(t^{\prime}\right) \in \eta^{\prime k_{i}}\left(p^{\prime}\right)\end{array}\right\}$,

- $Z=\left\{\left(p^{\prime},\left(x^{\prime a}\right),\left(y^{\prime b}\right)\right): \sum_{i \in \mathbb{N}} x^{\prime a_{i}}-\sum_{j \in \mathbb{N}} y^{\prime b_{j}}=\omega^{\prime}, x^{\prime a_{i}} \in \varphi^{\prime a_{i}}\left(p^{\prime}\right), y^{\prime b_{j}} \in \eta^{\prime b_{i}}\left(p^{\prime}\right)\right\}$,

- $\mu: E \rightarrow M$, where $\mu(e)=\left(m^{k_{1}}, m^{k_{2}}, \ldots\right)$,

- $h: M \rightarrow Z, h(m)=\left(p^{\prime},\left(x^{\prime a}\right),\left(y^{\prime b}\right)\right)$, where

$$
y^{\prime b} \stackrel{\text { def }}{=} Q\left(y^{b}\right), x^{\prime a} \stackrel{\text { def }}{=} x^{a}(t) .
$$

Due to conditions (4) and (6),

$$
\beta^{\prime a_{i}}(p)=\beta^{a_{i}}(p) \quad \text { and } \quad \varphi^{\prime a_{i}}(p)=\varphi^{a_{i}}(p) \text {, }
$$


for every $i \in \mathbb{N}$. Let, for every $j \in \mathbb{N}$,

$$
Y^{\prime b_{j}} \stackrel{\text { def }}{=} Q\left(Y^{b_{j}}\right) \text {. }
$$

Hence, for every $j \in \mathbb{N}$ and $y^{\prime} b_{j} \in Y^{\prime} b_{j}$, there exist $y^{b_{j}} \in Y^{b_{j}}$ such that

$$
y^{\prime b_{j}}=Q\left(y^{b_{j}}\right)=y^{b_{j}}-\left(g \circ y^{b_{j}}\right) \cdot \zeta
$$

as well as, for every $y^{\prime} b_{j} \in Y^{\prime} b_{j}$,

$$
\begin{gathered}
p \circ y^{b_{j}}=p \circ Q\left(y^{b_{j}}\right)= \\
=p \circ y^{b_{j}}-\left(g \circ y^{b_{j}}\right) \cdot(p \circ \zeta)=p \circ y^{b_{j}}-\left(\frac{p}{p \circ \zeta} \circ y^{b_{j}}\right) \cdot(p \circ \zeta)=0 .
\end{gathered}
$$

On the basis of the above, for every $j \in \mathbb{N}$,

$$
Q\left(y^{b_{j}^{*}}\right) \in \eta^{\prime b_{j}}(p)
$$

On the other hand, for every $i \in \mathbb{N}$,

$$
0=p \circ x^{a_{i}{ }^{*}} \leq p \circ \epsilon^{a_{i}}+\sum_{j \in \mathbb{N}} \theta\left(a_{i}, b_{j}\right) \cdot p \circ y^{b_{j}{ }^{*}}
$$

which gives that

$$
\sum_{j \in \mathbb{N}} \theta\left(a_{i}, b_{j}\right) \cdot p \circ y^{b_{j}^{*}} \geq 0
$$

For every $i \in \mathbb{N}$,

$$
\sum_{j \in \mathbb{N}} \theta\left(a_{i}, b_{j}\right) \cdot\left(p \circ Q\left(y^{b_{j}^{*}}\right)\right) \geq 0
$$

so, for $y^{\prime b_{j *}}=Q\left(y^{b_{j \star}}\right)$, the following is true

$$
0=p \circ x^{a_{i}^{*}} \leq p \circ \epsilon^{a_{i}}+\sum_{j \in \mathbb{N}} \theta\left(a_{i}, b_{j}\right) \cdot p \circ y^{\prime b_{j}^{*}} .
$$

Finally,

$$
\sum_{i \in \mathbb{N}} x^{\prime a_{i}^{*}}-\sum_{j \in \mathbb{N}} y^{b^{b_{j}^{*}}}-\omega=\sum_{i \in \mathbb{N}} x^{a_{i}^{*}}-\sum_{j \in \mathbb{N}} Q\left(y^{b_{j}^{*}}\right)-\omega=\mathbf{0} .
$$

On the basis of the above, the structure $\Gamma=(M, \mu, h)$ is the economic mechanism in the sense of Hurwicz resulting in equilibrium in economy $\mathcal{E}_{q}^{\prime}$. 
Condition (5) by the thesis of Theorem 1 means that the Walras Law is not satisfied for price vector $p$. Condition (4) means that total expenses with respect to the realization of every consumption plan and endowment in the considered economic system are equal to zero. Note that in (Mas-Colell et al., 1995, p. 335), the following remark can be found "A firm will enter the market if it can earn positive profits at the going market price and will exit if it can make only negative profits at any positive production level given this price (...) this implies that active firms must earn exactly zero profits in any long-run competitive equilibrium." If producers have zero profits then it makes sense to study zero worth consumers' processes. Especially if consumers' endowments are also worth zero at given prices. In the light of the cited Mas-Colell statement, the state of equilibrium defined in the proof of Theorem 1 is the longrun state of equilibrium.

Under assumptions (4), by Definition 3, we get that, for every $i \in \mathbb{N}$

$$
0=p \circ x^{a_{i}^{*}} \leq p \circ \epsilon^{a_{i}}+\sum_{j \in \mathbb{N}} \theta\left(a_{i}, b_{j}\right) \cdot p \circ y^{b_{j} *} .
$$

Hence as for every, every $j \in \mathbb{N}, \sum_{i \in \mathbb{N}} \theta\left(a_{i}, b_{j}\right)=1$,

$$
0=\sum_{i \in \mathbb{N}}\left(p \circ x^{a_{i}^{*}}\right) \leq p \circ \omega+\sum_{j \in \mathbb{N}}\left(p \circ y^{b_{j}^{*}}\right) .
$$

Combining (5), we obtain

$$
p \circ \zeta<0
$$

and

$$
\sum_{b \in B}\left(p \circ y^{b^{*}}\right)>0
$$

Condition (8) means that the maximal profit of at least one producer in economy $\varepsilon_{q}$ was greater than zero. The mechanism defined in the proof of Theorem 1 implies that, at time $t^{\prime}$, the maximal profit of every producer is equal zero. Hence mechanism $\Gamma$ defined in the proof of Theorem 1 does not change the economic position of consumers but the economic position of at least one producer became worse (see also Lipieta \& Malawski, 2016).

Notice that if, under assumptions of Theorem 1 , for every $j \in \mathbb{N}, Y^{\prime} b_{j}=Y^{b_{j}}$, then $P_{q} \subset_{i m} P_{q}^{\prime}$. If there exists $j \in \mathbb{N}$, for which $Y^{\prime} b_{j} \neq Y^{b_{j}}$, then $P_{q} \subset_{i n} P_{q}^{\prime}$. Hence the mechanism defined in the proof of Theorem 1 is either an innovation or an imitation mechanism. However, in both cases, it results in the equilibrium in economy $\varepsilon_{q}^{\prime}$. 
Theorem 1 confirms the validity of the model presented earlier ofSchumpeter's and Shionoya's thoughts. Under some initial conditions, after adapting innovations into routine activities of economic agents on the market, the economic system under study can achieve equilibrium as the consequence of an innovative or an imitative mechanism.

Using the methods by the proof of Theorem 1, the following theorem can be proved:

Theorem 2. Let $t, t^{\prime} \in\{0,1,2, \ldots\}, t<t^{\prime}$. If, at point $t$, in a private ownership economic $\varepsilon_{q}$ in which

$$
p \circ \zeta=0 \quad \text { and } \quad \zeta \neq \mathbf{0}
$$

there exists $q \in \mathbb{R}^{\ell}$ such that

$$
\forall a \in A: X^{a} \subset\left\{x \in \mathbb{R}^{\ell}: q \circ x=0\right\},
$$

then there exist an economic mechanism in the sense of Hurwicz, which transforms, at point $t^{\prime}$, economy $\varepsilon_{q}$ into an economy $\varepsilon_{q}^{\prime}$, in which a state of equilibrium at price $p^{\prime}=p$ exists as well as $C_{q} \subset_{i m} C_{q}^{\prime}, \ell=\ell^{\prime}, n=n^{\prime}, \epsilon\left(a_{i}\right)=\epsilon^{\prime}\left(a_{i}\right)$ and $\varepsilon\left(a_{i}\right)=\varepsilon^{\prime}\left(a_{i}\right)$, for every $i \in \mathbb{N}$.

Proof. Firstly, notice that by assumption (8) and (9), vector

$$
g \stackrel{\text { def }}{=} \frac{\zeta}{\zeta \circ \zeta} \in \mathbb{R}^{\ell}
$$

satisfies the following system of two equalities:

$$
\left\{\begin{array}{l}
g \circ \zeta=1 \\
g \circ p=0 .
\end{array}\right.
$$

Hence mapping

$$
Q: \mathbb{R}^{\ell} \ni x \rightarrow x-(g \circ x) \cdot \zeta \in \mathbb{R}^{\ell}
$$

is the linear and continuous projection into space $\left\{x \in \mathbb{R}^{\ell}: g \circ x=0\right\}$ (see Cheney, 1966). Moreover, for every $x \in \mathbb{R}^{\ell}$,

$$
p \circ Q(x)=p \circ x .
$$

Especially, for every $j \in \mathbb{N}$,

$$
p \circ Q\left(y^{b^{*}}\right)=p \circ y^{b_{j^{*}}},
$$

which means that production process $Q\left(y^{b_{j^{*}}}\right)$ maximize the profit of producer $b_{j}$ on set $Q\left(Y^{b^{*}}\right)$. In the above for every $i \in \mathbb{N}$ and $x^{a_{i}} \in \beta^{a_{i}}(p)$ 


$$
p \circ x^{a_{i}} \leq p \circ \epsilon^{a_{i}}+\sum_{j \in \mathbb{N}} \theta\left(a_{i}, b_{j}\right) \cdot p \circ Q\left(y^{b_{j}^{*}}\right),
$$

which gives that $\beta^{a_{i}}(p)=\beta^{\prime a_{i}}(p)$.

The rest of the proof goes in the same way as the proof of Theorem 1 .

Theorem 2 describes the situation when in a private ownership economy with prices $q$ satisfying condition (4), prices $q$ transform to prices given by vector $p$ satisfying condition (11), while the economic environment is not changed. Theorem 2 shows that there is a mechanism resulting in equilibrium in a transformation of the economy considered during which the economic positions of both producers and consumers are not changed. The mechanism determined in the proof of Theorem 2 is, as in case of Theorem 1 and for the same reason, either innovative or imitative.

Finally it should be emphasized that Theorems 1 and 2 only show that, under some initial conditions, disequilibrium prices can be equilibrium prices in an economic system with a transformed production sphere. The problem of specifying a mechanism resulting in equilibrium in the case where condition (4) is not satisfied remains still under study.

\section{Conclusions}

In this research production processes are divided into two categories: innovative and imitative. In the approach presented an economic mechanism can be seen as the set of production and consumption processes. An imitative mechanism is uniform, whereas every innovative mechanism contains imitative elements and at least one innovative production process. Such a setup enables a comparison of these two kinds of mechanisms with respect to their results.

Consider a group of economic mechanisms in which the set of environments and the set of outcomes form private ownership economies. The set of messages is equal to the set of outcomes as well as the outcome function being also equal to the identity function. As a result of the analysis presented the following can be concluded:

- if prices are not changed and a mechanism is imitative, then producers' profits might increase, but they do not exceed the maximal profit from the previous (initial) period,

- if prices are not changed and the mechanism is innovative, then producers' profits might be changed,

- if the mechanism is innovative then a new commodity or a new technology appears in the final economy with regard to the initial economy,

- if the mechanism is imitative then neither new commodities nor new technologies appear in the final economy with regard to the initial economy. 
Hence, it is easily seen that only an innovative mechanism could improve the economic position of some producers-innovators with respect to the previous period, if their profits increase. In an imitative mechanism, the profits can increase but they are not greater than profits from the earlier period.

As a result of innovative and imitative mechanisms, preferences, initial endowments and the share function might be changed, hence the economic positions of economic agents can be modified. They can be, but not necessarily, greater.

Additionally under some assumptions, the innovative as well the imitative mechanism can result in an equilibrium. The dilemma that finds no answer is the issue when with total expenses on consumption plans remaining non-zero and the Walras Law is not satisfied, could the equilibrium in the economic system considered still be achieved. On the basis of the above the conclusion is that evolutionary economic mechanisms are worth further study.

\section{References}

Acemoglu, D. (2009). Introduction to modern economic growth. Princeton and Oxford: Princeton University Press.

Adamowicz, Z. \& Zbierski, P. (1997). Logic of mathematics: A modern course of classical logic. New York: Wiley.

Aghion, P. \& Howitt, P. (1992). A model of growth through creative destruction. Econometrica, 60, 323-351.

Aghion, P.\& Howitt, P. (1998). Endogenous growth theory. Cambridge and London: MIT Press.

Arrow, K. J . \& Debreu, G. (1954). Existence of an equilibrium for a competitive economy. Econometrica 22, 265-290.

Bessen, J. \& Maskin, E. (2009). Sequential innovation, patents, and imitation. Journal of Economics, 40(4), 611-635. https://doi.org/10.1111/j.1756-2171.2009.00081.x

Bolton, M. K. (1993). Imitation versus innovation. Lessons to be learned from the Japanese. Organizational Dynamics, 21(3), 30-45. https://doi.org/10.1016/00902616 (93)90069-d

Cantner, U. (2016). Foundations of economic change-an extended Schumpeterian approach. Journal of Evolutionary Economics, 26, 701-736. doi: 10.1007/s00191016-0479-z

Cheney, E. W. (1966). Introduction to approximation theory. New York: McGraw Hill.

Ciałowicz, B. \& Malawski, A. (2011). The role of banks in the Schumpeterian innovative evolution-an axiomatic set-up. In: A. Pyka, F. Derengowski \& M. da Graca (Eds.), Catching up, spillovers and innovations networks in a Schumpeterian perspective (pp. 31-58). Springer.

Ciałowicz, B. \& Malawski, A. (2016). The logic of imitative processes: imitation as secondary Innovation - an axiomatic Schumpeterian analysis. Argumenta Oeconomica Cracoviensia, 15, 43-56. doi: 10.15678/AOC.2016.1503

Debreu, G. (1959). Theory of value. New York: Wiley. 
Glass, A. J. (2010). Imitation as a stepping stone to innovation. College Station: Department of Economics, Texas A\&M University.

Herrmann-Pillath, C. (2013). Foundations of economic evolution. Cheltenham, Northampton, Massachusetts: Edward Elgar.

Hurwicz, L. (1987). Incentive aspects of decentralization. In: K. J. Arrow, M. D. Intriligator (Eds.), Handbook of mathematical economics, vol. 3. Amsterdam, Netherlands.

Hurwicz, L. \& Reiter, S. (2006). Designing economic mechanism. New York: Cambridge University Press.

Lipieta, A. (2013). Mechanisms of Schumpeterian evolution. In: A. Malawski (Ed.), Innovative economy as the object of investigation in theoretical economics (pp. 94119). Cracow: Cracow University of Economics Press.

Lipieta, A. \& Malawski, A. (2016). Price versus quality competition: In search for Schumpete-rian evolution mechanisms. Journal of Evolutionary Economics, 26(5), 1137-1171. doi:10.1007/s00191-016-0470-8.

Lipieta, A. \& Malawski A. (2018). Comparative analysis of mechanisms of Schumpeterian evolution. Journal of Entrepreneurship, Management and Innovation, 14, 7-28.

Mas-Colell, A., Whinston, M. D. \& Green J. R. (1995). Microeconomic theory. Oxford: Oxford University Press.

Mukoyama, T. (2003). Innovation, imitation, and growth with cumulative technology. Journal of Monetary Economics, 50(2), 361-380. https://doi.org/10.1016/s03043932(03)00005-9.

Nelson, R. (2016), Behavior and cognition of economic actors in evolutionary economics. Journal of Evolutionary Economics, 26, 737-751. doi: 10.1007/s00191-0150431-7.

Nelson, R. R., \& Winter, S. (1982). An evolutionary theory of economic change. Cambridge, Mass.: Belknap Press of Harvard University Press.

Romer, D. (2012). Advanced macroeconomics (4th edition). New York: McGraw-Hill.

Rossi, J. P. (2003). Innovation, imitation and entrepreneurship; the introduction and diffusion of the homeowners policy, 1944-1960. In: Essays in Economic and Business History (vol. XXI), published by the EBHS and Department of History the University of Mississippi. www.ebhsoc.org/journal/index.php/journal/article/ viewFile/104/991.

Safarzyńska, K. \& van den Bergh, J. C. J. M. (2010). Evolutionary models in economics: A survey of methods and building blocks. Journal of Evolutionary Economics, 20(3), 329-373. https://doi.org/10.1007/s00191-009-0153-9

Schumpeter, J. A. (1912). Die Theorie der wirtschaftlichen Entwicklung. Leipzig: Duncker \& Humblot. English translations: The theory of economic development, Cambridge: Harvard University Press 1934 and A Galaxy Book, New York, Oxford University Press 1961.

Schumpeter, J. A. (1964). Business cycles: A theoretical, historical, and statistical analysis of the capitalist process. New York, London: McGraw-Hill.

Segerstrom, P. S. (1990). Innovation, imitation and economic growth. Econometrics and Economic Theory Paper No. 8818. East Lansing: Michigan State University

Shenkar, O. (2010, April). Defend your research: Imitation is more valuable than innovation. Harvard Business Review, 28-29. 
Shionoya, Y. (2007). Schumpeterian universal social science. In: H. Hanusch., A. Pyka (Eds.), Elgar Companion to Neo Schumpeterian Economics (pp. 55-65). Cheltenham, Northampton, MA: Edward Elgar.

Simon, H. A. (1955). A behavioural model of rational choice. The Quarterly Journal of Economics, 69(1), 99-118. 